\title{
Optimization Study for Excitation System Simulation Model with Limit Function
}

\author{
Qian $\mathrm{CHENG}^{1}$, Junfeng $\mathrm{ZHANG}^{2}$, Cong $\mathrm{FU}^{2}$ \\ ${ }^{1}$ School of Electrical Engineering, Northeast Dianli University, Jilin, 132012, China \\ ${ }^{2}$ Electric Power Research Institute, Guangdong Power Grid Corporation, Guangzhou, 510080, \\ China \\ aemail: 13610767831@163.com
}

\begin{abstract}
Keywords: Power System Simulation; Terminal Voltage; Optimal Modeling; RTDS
\end{abstract}
\begin{abstract}
In power system analysis software the simulation model of generator excitation system is not perfect, actual characteristics of the generator cannot be simulated accurately under some certain faults. Thus, an improved excitation simulation model with excitation limit is presented in this paper. The model structure and principle of excitation limit is studied and through using custom a module of DSP the improved excitation system is modeled. Then establish a single-machine infinite-bus system model in RTDS and PSD-BPA to verify the correctness and effectiveness of the simulation model. Finally through the comparison of dynamic simulation results of Guangdong grid, the method proposed in this paper effectively limits the terminal voltage.
\end{abstract}

\section{Introduction}

Generator is an important reactive power of the system. Voltage sags due to system failures; the reactive power of the unit to the system is divided into two parts: The first part is set as the power supply voltage and the system voltage exist difference, the unit outputs reactive power instantaneously. The second part is set excitation system (constant terminal voltage operation mode) will force excitation to increase excitation current to provide more dynamic reactive power support when the terminal voltage drops[1]. Rational control of reactive power supply is an important measure to ensure the stability and voltage level of the system [2][3]. The excitation system provides the excitation power for the generator, which has the function of regulating voltage and keeping the terminal voltage constant. Besides it can control the reactive power distribution of the parallel running generator. It has a great influence on the dynamic operation of the generator, which can improve the stability limit of the power system[4][5]. So it is very important to establish the correct excitation system model in the simulation of power system[6][7].

Generator excitation control must meet the corresponding constraints, which is an important part of the voltage constraint[8]. In order to maintain the safe and stable operation of the generator, related standards of excitation regulator set some necessary restrictions to prevent the generator from exceeding the output range of the design and the damage to the equipment. Modern generator excitation has a comprehensive protection system, which includes the minimum excitation limit, over excitation limit, voltage / frequency limit, terminal voltage limitation, reactive power control and PT disconnection protection, etc[9][10][11]. High or low voltage of the generator may lead to protective action, which can affect the control effect and even lead to the control failure, which affects the operation safety of the system. Therefore, the limit of the protection of the terminal voltage is an inevitable problem. Under the premise of ensuring the stability of the system, the limit of terminal voltage is worth to be studied in depth[12].

The existing power system simulation software provides a variety of mathematical models of the generator excitation system, not including all the excitation system in the practical application. And with the development of the power technology and the input of the new control device, the standard model library has been unable to meet the requirements. In actual power grid simulation; the terminal voltage of the generator exceeds its limit due to the inaccurate model of the excitation system model. In this paper, a new excitation system model with top value constraint is established, 
in view of the voltage limitation which exits in a long-time and low-voltage serious fault.

Add excitation limit to excitation system of the generator which may occur voltage limitation, and study the response of the simulation model of the excitation system with excitation limit in grid voltage dip period.

\section{Analysis of existing models}

In the field of domestic power grid planning, PSD-BPA power flow stability calculation software is widely used as a support tool for the quantitative calculation of power grid. Through a large number of experimental simulations of power grid, we find that generator output will grow unrestrictedly, even more than several times the rated capacity, and the terminal voltage will exceed 1.1 times the system rated voltage of GB as well, under the circumstance of continuous low voltage of load end. The reliability of simulation results will be greatly reduced, even affecting the critical judgment of the system stability.

Take three phase short circuit single phase switch refusal in Beijiao station $(50 \%$ proportional motor model of Guangdong Power Grid in 2013) as an example, to simulate by using BPA(Version number: 4.2). The continuous low voltage characteristic of the load end is shown in Fig. 1.

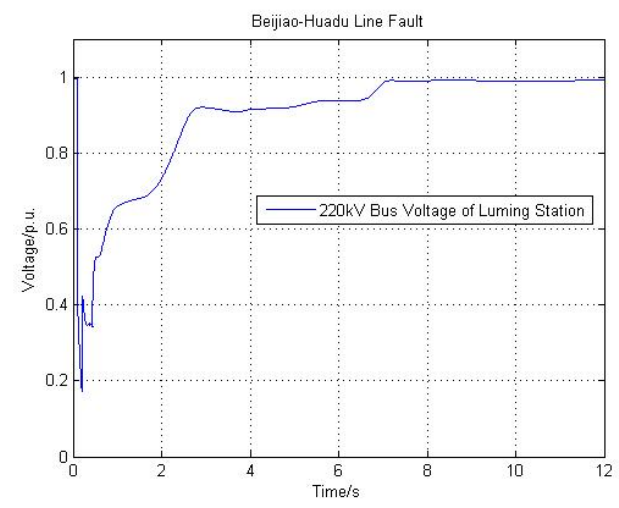

Fig. 1. Bus voltage curve of LuMing station

Figure 1 shows that there is an apparent voltage delay recovery problem in $220 \mathrm{kV}$ Luming Station after fault. After power grid disturbance, some bus voltage will drop and then the generator (near the bus) should quickly start reactive power control to compensate the shortage of reactive power in system[13]. Unit 1 in Guangzhou Pumped Storage Power Station as a power source near Beijiao, the voltage and reactive power output of the machine is shown in Figure 2 and Figure 3.

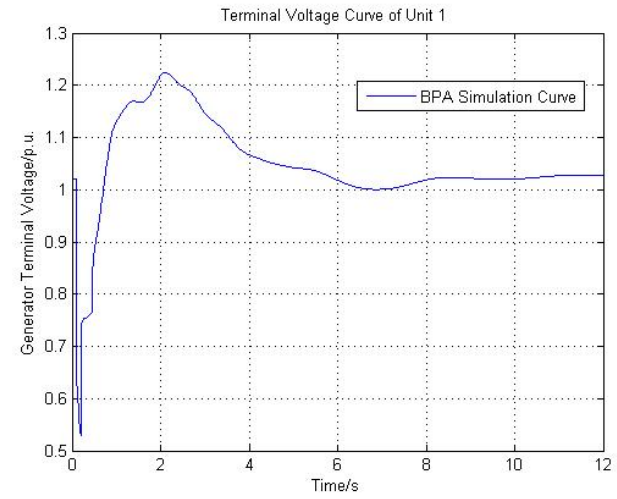

Fig. 2. Terminal voltage curve of faulted unit lin Guangzhou Pumped Storage Power Station 


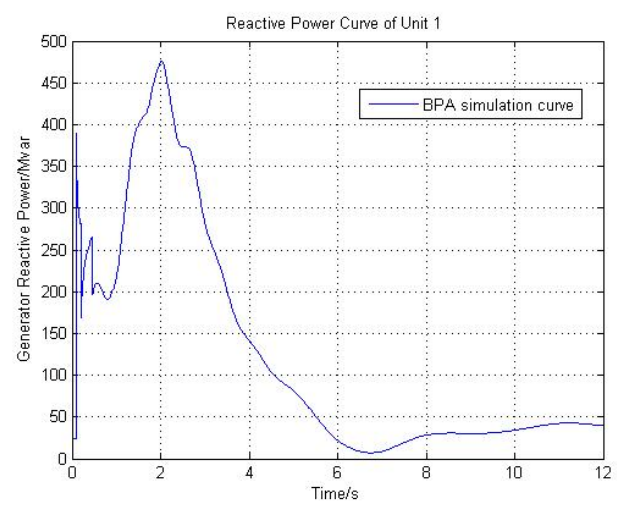

Fig. 3. Reactive power curve of faulted unit 1 in Guangzhou Pumped Storage Power Station

Simulation results show that the terminal voltage of unit 1 in Guangzhou Pumped Storage Power Station is 1.2 times of the rated value and reactive power far exceed the rated apparent capacity. The terminal voltage and reactive power grow unrestrictedly, with generator serious overload, which differ greatly from the actual operating conditions. The simulation result is distorted. According to the "technical guidelines for power system voltage and reactive power" and "the technical requirements for turbine type synchronous motor", the terminal voltage of generator has exceeded the standard of "After system disturbance, the bus voltage allowable deviation value of the power plant and substation bus cannot exceed $\pm 10 \%$ of the rated voltage of the system"[14][15].

The main purpose of the excitation regulator is to control and regulate the terminal voltage of the synchronous generator. Through the analysis, it is known that the excitation system model is not perfect, due to the simulation software failed to accurately simulate the actual generator external characteristic. Overexcitation phenomenon exists in simulation, and we cannot achieve the purpose of restraining the voltage of the machine by regulating the excitation. So, it should be considered voltage limit and overvoltage protection.

\section{Model and principle of excitation limit}

\section{VFL Model introduction}

The peak value limit of generator excitation is an important nonlinear part in power system[16]. Excitation regulator as the control center of excitation system, with the development of modern control theory and the application of computer technology, the excitation regulator is mainly based on digital controller at present. The VFL model for the time delay action or the inverse time limit is shown in Figure 4. The model is modified and simplified by reference to a number of manufacturers' equipment, which has a certain representative significance.

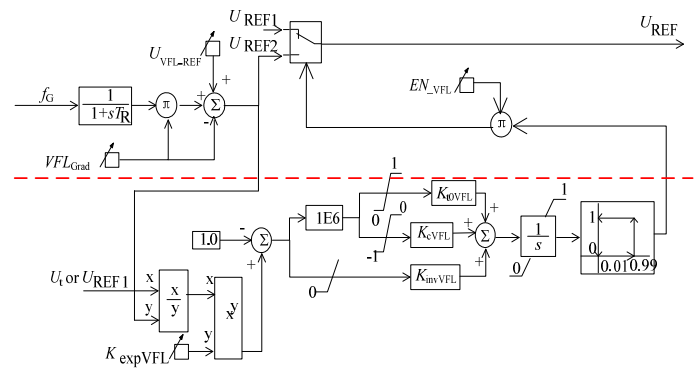

Fig. 4. VFL model of deferred action or inverse-time

The model is mainly divided into two parts. One is the voltage output link, specific parameter: $U_{\mathrm{VFL}} \mathrm{REF}$ is VFL action reference value; $V F L_{\mathrm{Grad}}$ is VFL characteristic slope; $f_{\mathrm{G}}$ is input frequency; $U_{\mathrm{t}}$ is input terminal voltage; $U_{\mathrm{REF} 1}$ is AVR reference voltage; $U_{\mathrm{REF}}$ is output voltage.

The other is inverse time limit, specific parameter: $T_{\mathrm{R}}$ is measuring time constant; $T_{\mathrm{I}, \mathrm{VFL}}$ is integral time constant; $K_{\text {expVFL }}$ is inverse time characteristic index coefficient; $K_{\text {toVFL }}$ is fixed time integral constant, which is the reciprocal of the fixed time; $K_{\text {invVFL }}$ is inverse-time characteristic integral constant; $K_{\mathrm{cVFL}}$ is cooling integral time constant. 
Model output uses the "comparative gate" way to intervene in the main loop of voltage control, where $E N_{-V F L}$ is the input license switch of VFL, 1- input, 0- exit. For example, if the generator frequency $f_{\mathrm{G}}$ is reduced after the system disturbance, the frequency variation $\Delta f_{\mathrm{G}}<0$, then the switch $E N_{\text {-VFL }}$ act and VFL input. $U_{\text {REF2 }}$ is reduced after excitation limit, and less than $U_{\text {REF1 }}$. The smaller one of $U_{\text {REF1 }}$ and $U_{\text {REF2 }}$ will be used as the output voltage $U_{\text {REF }}$.

$$
U_{\mathrm{REF}}= \begin{cases}U_{\mathrm{REF} 1}, & \Delta f_{\mathrm{G}} \geq 0 \\ U_{\mathrm{REF} 2}, & \Delta f_{\mathrm{G}}<0\end{cases}
$$

\section{Single machine infinite bus model considering excitation limitation}

In order to prevent the generator from over voltage and endanger the safety of the unit, the excitation regulator is set up with the terminal voltage limit and over voltage protection function unit. Prevent it from continuing to increase the generator excitation current, when the terminal voltage reaches or exceeds the limit. The $\mathrm{V} / \mathrm{Hz}$ limit is the ratio limit of voltage and frequency generator, preventing the generator and main transformer overexcitation.

The generator voltage is known to be proportional to the number of turns of the winding and the flux of the chain, i.e. $U_{t}=4.44 f W \Phi(f$ is the system frequency). Since turns are fixed, so can be obtained:

$$
\phi=\frac{U_{t}}{4.44 f W}
$$

By formula (1) it is known that the flux is proportional to the ratio of voltage and frequency. Voltage rise or frequency drop will cause the increase of the magnetic flux, which will cause over magnetic flux of the generator. And the magnetic flux of transformer connected with the generator will increase, resulting in winding, iron core overheating, and increasing eddy current losses, damage to equipment insulation.

The expression of overexcitation multiple $\mathrm{n}$ is shown in the formula (2):

$$
\mathrm{n}=\frac{\phi}{\phi_{\mathrm{N}}}=\frac{U / f}{U_{\mathrm{N}} / f_{\mathrm{N}}}
$$

Where $U_{N}$ is the rated voltage of the unit; $f_{N}$ is the system rated frequency. $\mathrm{n}$ is generally about 1 to 1.5 , representing the severity of overexcitation of generator. $\mathrm{V} / \mathrm{Hz}$ limit can suppress this situation, preventing unit voltage rise or frequency decrease. When the generator is connected to the grid, because the generator frequency is the frequency of the system, the actual $\mathrm{V} / \mathrm{Hz}$ limit is mainly terminal voltage limit.

Limiting link is generally set in the generator excitation system regulator. With real-time digital simulation system RTDS(real time digital simulator) as the simulation platform, a single machine infinite bus system with excitation limit is set up to simulate the limiting effect of the actual controller device. Specific simulation results will be presented in the third section of this paper. The specific location of $\mathrm{V} / \mathrm{Hz}$ limit in a single machine infinite bus system is shown in Figure 5[17].

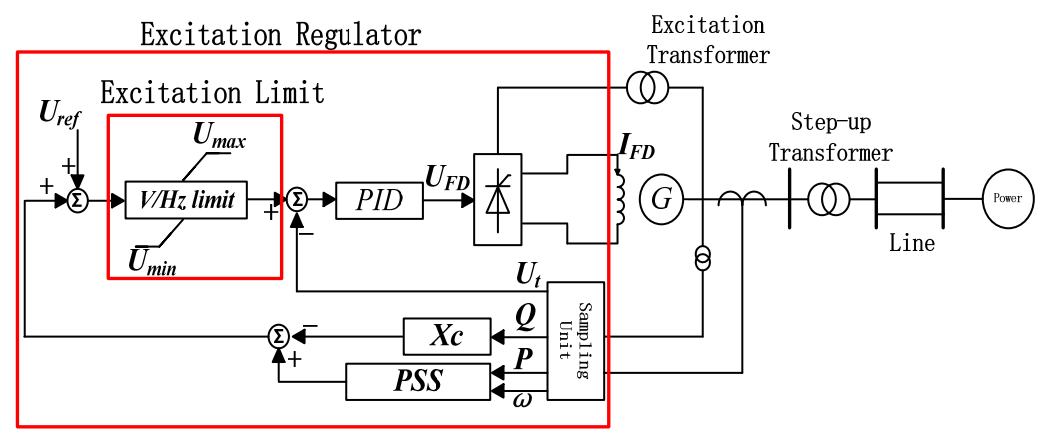

Fig. 5.Excitation limits considered single machine infinite bus system

Where $U_{\text {ref }}$ is taken as voltage given value, $U_{\mathrm{FD}}$ is excitation voltage, $U_{\max }$ is excitation limit top voltage, PID is the excitation system of integral proportion differential control method, $I_{\mathrm{FD}}$ is excitation current, $U_{t}$ is terminal voltage, $\mathrm{P}$ is active power, $Q$ is reactive power, $\omega$ is generator speed, $X_{\mathrm{C}}$ is adjustment coefficient. 
It can be seen from the model that the excitation limit not only affects the value of the given value of the terminal voltage $U_{\text {ref, }}$ it also affects the effect of the differential coefficient $X_{\mathrm{c}}$. For negative adjustment, with the increase of generator reactive power output, the terminal voltage will always be greater than a given value. In order to limit the machine voltage, prevent damage to equipment insulation, excitation limit has played a key role.

\section{Simulation of single machine model}

According to the above description, this paper establishes a model of the improved generator excitation system with the consideration of the peak value of the excitation. Follow up study with the aid of AC / DC hybrid power system electromechanical and electromagnetic hybrid simulation software CSGDSP (referred to as DSP), which is researched independently by China Southern Power Grid Science Research Institute, to carry on the example model to improve the description. The design and development of DSP is mainly based on BPA cards, which can be used to define various kinds of electrical components and control models based on the specific text format. DSP has a high degree of scalability, allowing users to follow the specified rules to define their own specific new module. Custom modeling flow chart is shown in Figure 6.

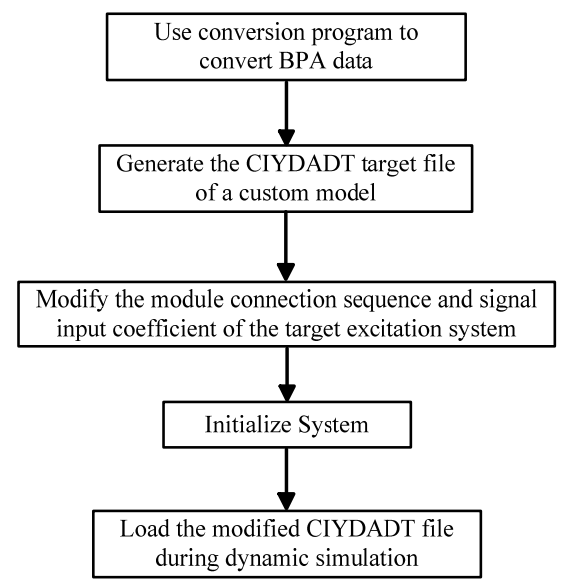

Fig. 6.Compilation process of user defined model

In order to verify the correctness of the improved model of excitation system, a single machine infinite bus system considering the limitation of excitation, the same as RTDS, is built by using DSP. Set the same fault to compare the simulation curves. In RTDS simulation system, the actual excitation regulator device is used to realize the closed loop control while in DSP use an improved excitation model with custom built. Set $50 \Omega$ three-phase line-to-ground fault at the high voltage side of the main transformer in two simulation platform at the same time, to monitor the terminal voltage and the generator reactive power output, and verify the limit function and the action characteristic of the excitation regulator. At present, the voltage limiting action of the unit is generally taken as: 1.06 1.08p.u. The starting value of the actual field excitation regulator, connected to the RTDS, is 1.1 . Therefore, set the action limit value of the terminal voltage to 1.1 p.u.. The specific parameters of the excitation system of a single machine infinite bus model are shown in Table 1.

Tab. 1. Excitation system parameters of single machine infinite bus model

\begin{tabular}{|c|c|}
\hline Model & Excitation system parameters \\
\hline \multirow{3}{*}{ FU } & $\begin{array}{c}\mathrm{K}=480, \mathrm{~K}_{\mathrm{V}}=1, \mathrm{~K}_{\mathrm{A}}=1, \mathrm{~K}_{\mathrm{F}}=0, \mathrm{~K}_{\mathrm{C}}=0, \mathrm{~T}_{\mathrm{R}}=0.02 \mathrm{~s} \mathrm{~T}_{1}=1 \mathrm{~s}, \\
\end{array}$ \\
$\begin{array}{r}\mathrm{T}_{2}=10 \mathrm{~s}, \mathrm{~T}_{3}=1 \mathrm{~s}, \mathrm{~T}_{4}=1 \mathrm{~s}, \mathrm{~T}_{\mathrm{A}}=0.01 \mathrm{~s}, \mathrm{~T}_{\mathrm{F}}=100 \mathrm{~s}, \mathrm{~V}_{\mathrm{AMAX}}=50, \\
\mathrm{~V}_{\mathrm{AMIN}}=-50, \mathrm{~V}_{\mathrm{RMAX}}=10, \mathrm{~V}_{\mathrm{RMIN}}=-10\end{array}$ \\
\hline
\end{tabular}

In addition, the model of single machine infinite bus system with the same structure and parameters is built in BPA, to serve as a reference group for this simulation by setting the same short-circuit fault. FU rotating excitation system is adopted in the model. The simulation results of a single machine infinite bus system are shown in Fig. 7 and Fig. 8. 


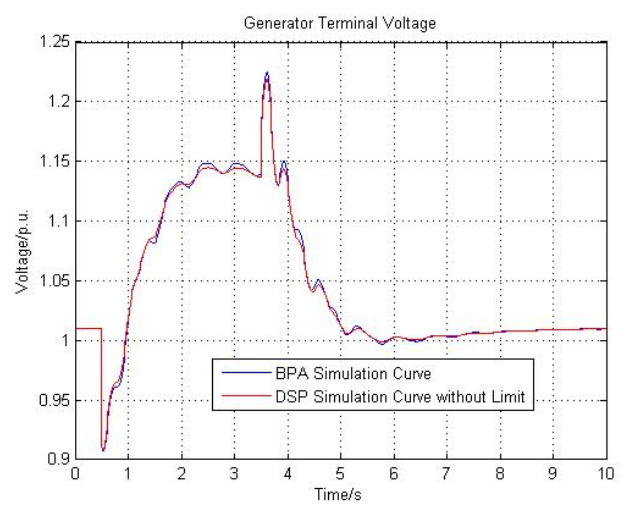

Fig. 7. Terminal voltage simulation comparison between BPA and DSP (before modifying excitation model)

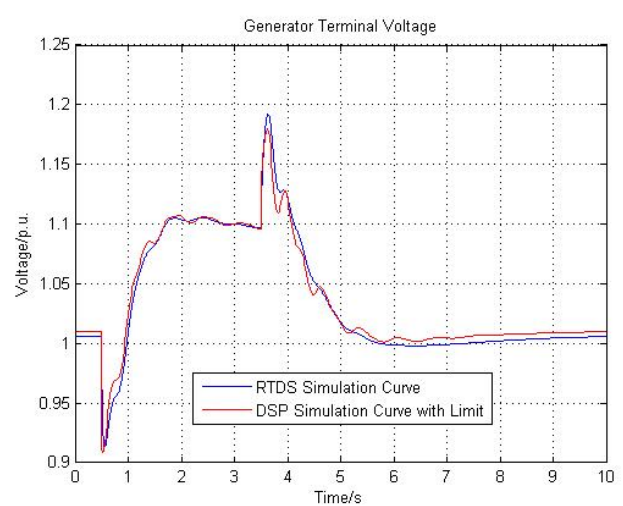

Fig. 8. Terminal voltage simulation comparison between RTDS and DSP (after modifying excitation model)

The simulation results show that the excitation limit is usually set in the actual excitation regulation device. The limit is added to the improved DSP model, which can effectively simulate the actual operation state of the excitation regulator. Taking the BPA results as the simulation control, due to the generator excitation system model has not yet considering the excitation limit, leading to a certain error between the results of the simulation and the actual excitation regulator.

The above examples can be concluded as follows:

(1) According to the simulation results, it can be seen that in the absence of an improved excitation system model, the DSP simulation results are in agreement with the BPA simulation results, and there are the distortion of the unit simulation results;

(2) DSP has a custom modification and modeling capabilities, which allows us to use its custom function to improve the simulation of the current simulation model for optimization;

By using the improved excitation model, the simulation curves of DSP and RTDS (generator excitation is provided by the actual excitation regulator) are in good agreement, which verifies that the improved model can accurately simulate the dynamic simulation process of the generator excitation system.

\section{Simulation analysis of multi machine system}

To further verify the correctness of the custom of excitation system model, this section still use Guangdong power grid in 2013 as an example to verify the simulation results.

Fault setting specific circumstance is three phase short circuit faults occurred in Beijiao side of Beijiao-Huadu $500 \mathrm{kV}$ line at $0.1 \mathrm{~s}$, the switching single phase rejection, and then the other circuit trip at $0.45 \mathrm{~s}$. The example load uses $50 \%$ induction motor $+50 \%$ constant impedance model. In order to improve the sensitivity of the generator to the power grid voltage, the unit adopts $10 \%$ negative adjustment, i.e. setting the adjustment coefficient $X_{\mathrm{c}}$ of the relevant units to $-0.1[18][19]$.

The Figure 2, Figure 3 shows that the terminal voltage of the Unit 1 in Guangzhou Pumped 
Storage Power Station voltage exceeds its limit. Convert the BPA data of the actual system of Guangdong power grid into DSP data. In DSP, the generator uses the improved excitation model, FV static self and shunt excitation system. After the excitation limit is added to excitation system, the new model is simulated and verified by the DSP simulation platform. And compare the simulation results with the case without excitation limit. Fig. 9 and Fig. 10 are simulation results comparing curves.

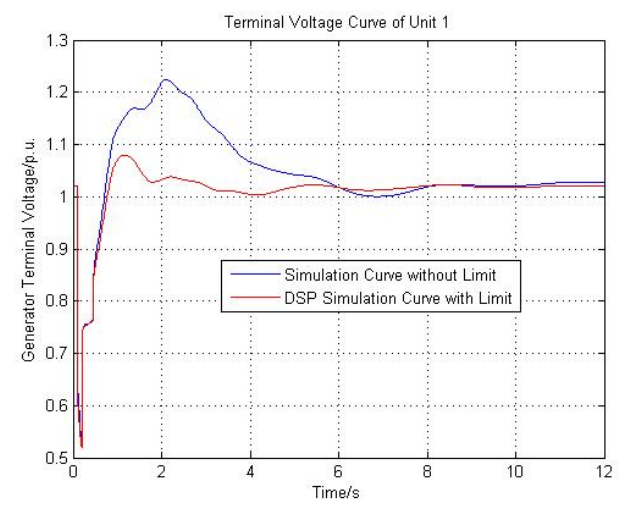

Fig. 9. Terminal voltage curve (after modifying excitation model) of unit 1 in Guangzhou Pumped Storage Power Station with excitation limit

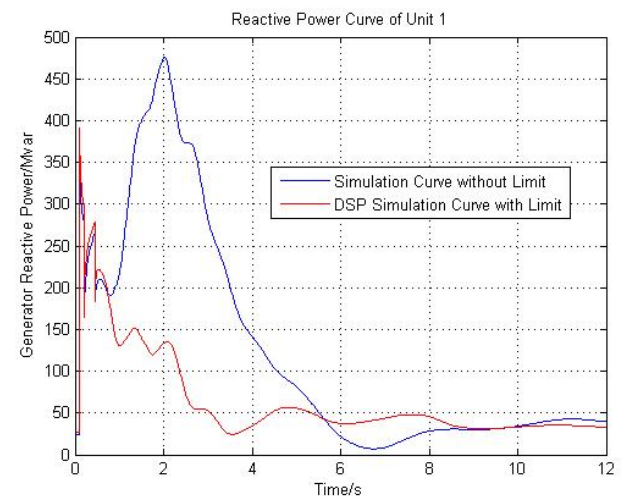

Fig. 10. Reactive power curve (after modifying excitation model) of unit 1 in Guangzhou Pumped Storage Power Station with excitation limit

Compared the simulation results of the improved excitation system model with the original simulation results, it can be obtained that there is an obvious limitation of the terminal voltage of the generator unit in the absence of the improved excitation system model, and the generator is in serious overload. After the model of the excitation system is improved, the reactive power and voltage of the generator is effectively controlled. High reliability of simulation results further show that the proposed excitation system model is correct. Generator excitation limit is mainly to prevent the generator over excitation, for units in grid-connected operation, mainly is to prevent the unit overvoltage. Excitation limit has important significance for voltage recovery after disturbance of the system.

\section{Conclusions}

In view of the existing power system analysis software may cause the generator terminal voltage limit Under the serious fault of the power grid, the reason of the distortion of the simulation result is analyzed, and then the model of the automatic excitation regulator is improved. By using DSP custom module, an improved excitation simulation model with constraint function is established to improve the simulation performance of the unit under a long-time and low-voltage fault. The accuracy of the model is verified by comparing with the simulation results of RTDS, which is combined with the actual field excitation regulator panel. In the actual production data, the model is 
used to calculate and compare with the results before the improvement. The results show that the proposed method can effectively restrain the terminal voltage under the severe faults.

\section{References}

[1] Shizhang Zhu. Test for Generator Excitation System [M]. Beijing: China Electric Power Press, 2005: 45-46.

[2] IBA K. Reactive power optimization by genetic algorithm [J]. IEEE Trans on Power Systems, 1994, 9(2): 685-692.

[3] Shuangxi Zhou, Lingzhi Zhu, Xijiu Guo, etc. Voltage Stability and Control of Power System[M]. Beijing: China Electric Power Press, 2009.

[4] Guiping Zhu, Shumin Wang. A survey on power quality control technology[J]. Automation of Electric Power Systems, 2002, 26(19): 28-31.

[5] Yixin Ni, Shousun Chen, Baolin Zhang, etc. Theory and Analysis of Dynamic Power System[M]. Beijing: Tsinghua University Press, 2002: 61.

[6] Yong Tang, Wuzhi Zhong, Huadong Sun. Study on Mechanism of Power System Voltage Stability [J].Power System Technology, 2010, 34(4): 24-29.

[7] Yong Tang. A discussion about standard parameter models of synchronous machine [J]. Power System Technology, 2007, 31(12): 47-51.

[8] Li Wan, Changhong Deng, Yunping Chen. Multi-layer Nonlinear Variable Structure Excitation Control Considering Voltage Considering Voltage Limitation[J]. Proceeding of the CSEE, 2008, 28(29): 86-92.

[9] Qu Liu. Stability of Power System and Generator Excitation Control[M]. Beijing: China Electric Power Press, 2007.

[10]DL/T684-1999, Setting Calculation Guide of Relay Protection for Large Generator Transformer [S].

[11]Jianjun Zhao, Considerable problems in setting protection unit set points of generator excitation control systems [J].North China Electric Power,2006,35(11): 44-47.

[12] Yingduo Han, Xiaorong Xie, Wenjin Cui. Status quo and future trend in research on synchronous generator excitation control [J].Tsinghua Univ. (Sci. \&Tech.), 2001, 14(4/5): 142-146.

[13] Jingxing Tang, Wen Sun, Xiaoming Li. Dynamic Reactive Power Control Strategy of Generators Based on WAMS [J].Southern Power System Technology, 2015(7): 82-87.

[14] SD325-89, Technical Guidelines for Voltage and Reactive Power in Power System [S].

[15]GB/T7064-2002, Technical Requirements for Turbine Type Synchronous Motor [S].

[16] Hui Shu, Jinyu Wen, Chunfeng Luo, et al. Nonlinear Parameters Identification for Synchronous Generator Excitation Systems [J]. Automation of Electric Power Systems, 2005, 29(6): 66-70.

[17]Junfeng Zhang, Peng Li, Yinsheng Su, et al. Risk Analysis on Optimization and Setting for System Reactive Current Compensation [J]. Automation of Electric Power Systems, 2015, 39(20):141-145.

[18]Jun An, Gang Mu, Taiyi Zheng. Optimization Strategy for Generation Excitation System Adjustment Coefficient in Improving Voltage Level of Power System[J]. Proceedings of the EPSA, 2013, 37(23): 97-101.

[19] Yuanzhang Sun, Yu Jia, Lin Cheng, et al. Optimum design of load compensation coefficient in excitation control [J].Proceeding of the CSEE, 2006, 26(23): 20-24. 